\title{
Inequalities for the $s$ th Derivative of A Polynomial with Prescribed Zeros
}

\author{
M.S. Pukhta \\ Division of Agri. Statistics, Sher-e-Kashmir University of Agricultural Sciences and Technology of Kashmir, 191121, India \\ ${ }^{*}$ Corresponding Author: mspukhta_67@yahoo.co.in
}

Copyright $\odot 2013$ Horizon Research Publishing All rights reserved.

\begin{abstract}
Let $p(z)$ be a polynomial of degree $n$ which does not vanish in $|z|<k, k \geq 1$, then for $1 \leq R \leq k$ Bidkham and Dewan [J. Math. Anal. Appl. 166 (1992), 191-193] proved

$$
\max _{|z|=R}\left|p^{\prime}(z)\right| \geq \frac{n(R+k)^{n-1}}{(1+k)^{n}} \max _{|z|=1}|p(z)| .
$$

In this paper we shall present several intersting generalizations and a refinement of this result which includes some results due to Malik, Govil and others. We also present a refinement of some other results.
\end{abstract}

Keywords Derivative of a polynomial, Zeros, Inequalities

MSC (2010) 30A10, 30C10.

\section{Introduction}

For $p \in p_{n}$ where $p_{n}$ be class of polynomials $p(z)$ of degree atmost $n$, we define

$$
\begin{aligned}
\|p\|_{\gamma} & :=\left\{\frac{1}{2 \pi} \int_{0}^{2 \pi}\left|p\left(e^{i \theta}\right)\right|^{\gamma}\right\}^{\frac{1}{\gamma}}, \quad 1 \leq \gamma<\infty \\
\|p\|_{\infty} & :=\max _{|z|=1}|p(z)| \quad \text { and } \\
m & :=\min _{|z|=k}|p(z)| .
\end{aligned}
$$

Let $p(z)$ be a polynomial of degree $n$, then according to a famous result known as Bernstein inequality (for reference see [13] or [12])

$$
\max _{|z|=1}\left|p^{\prime}(z)\right| \leq n \max _{|z|=1}|p(z)| .
$$

The result is best possible and equality holds for the polynomial having all its zeros at origin.

If we restrict ourselves to the class of polynomials having no zeros in $|z|<1$, then inequality (1.1) can be replaced by

$$
\max _{|z|=1}\left|p^{\prime}(z)\right| \leq \frac{n}{2} \max |p(z)| .
$$

As an extension of (1.2) Malik [8] verified that if $p(z)$ does not vanish in $|z|<k, k \geq 1$, then

$$
\max _{|z|=1}\left|p^{\prime}(z)\right| \leq \frac{n}{1+k} \max _{|z|=1}|p(z)| \text {. }
$$

Equality holds for $p(z)=(z+k)^{n}$.

Bidkham and Dewan [2] obtained a generalization of (1.3) for the same class of polynomials by proving

$$
\max _{|z|=R}\left|p^{\prime}(z)\right| \geq \frac{n(R+k)^{n-1}}{1+k} \max _{|z|=1}|p(z)| .
$$

The result is best possible and equality holds for $p(z)=$ $(z+k)^{n}$.

In the reverse direction it was proved by Turan [9] that if $p(z)$ does not vanish in $|z|>1$, then

$$
\max _{|z|=1}\left|p^{\prime}(z)\right| \geq \frac{n}{2} \max _{|z|=1}|p(z)| .
$$

Inequality (1.5) was refined by Aziz and Dawood [1] by showing that under the same hypothesis that

$$
\max _{|z|=1}\left|p^{\prime}(z)\right| \geq \frac{n}{2}\left\{\max _{|z|=1}|p(z)|+\min _{|z|=k}|p(z)|\right\} .
$$

Both the inequalities (1.5) and (1.6) are sharp and equality holds for $p(z)=\alpha z^{n}+\beta$, where $|\alpha|=|\beta|$. As an extension of (1.5), Govil [3] proved that if $p(z)$ is a polynomial of degree $n$ having all its zero in $|z| \leq k, k \leq 1$, then

$$
\max _{|z|=1}\left|p^{\prime}(z)\right| \geq \frac{n}{1+k} \max _{|z|=1}|p(z)|
$$

Equality holds for $p(z)=(z+k)^{n}, k \leq 1$.

Whereas if $p(z)$ has all its zeros in $|z| \leq k, k \leq 1$ with $s$-fold zeros at the origin, then Aziz and Shah [4] proved that

$$
\max _{|z|=1}\left|p^{\prime}(z)\right| \geq \frac{n+s k}{1+k} \max _{|z|=1}|p(z)|
$$

The result is sharp and extremal polynomial is $p(z)=$ $z^{s}(z+k)^{n-s}, 0<s \leq n$. 
Govil and Mctume [6] generalized inequality (1.6) of Aziz and Dawood [1] by proving that if $p(z)$ has all its zeros in $|z| \leq k, k \leq 1$, then

$$
\max _{|z|=1}\left|p^{\prime}(z)\right| \geq \frac{n}{1+k}\left\{\max _{|z|=1}|p(z)|+\frac{1}{k^{n-1}} \min _{|z|=k}|p(z)|\right\} \text {. }
$$

The result is best possible and equality holds for the polynomial $p(z)=(z+k)^{n}$.

\section{Main Results}

In this paper we shall first present the following generalization as well as an improvement of (1.4) by considering the $s$ th derivative of $P(z)$. More precisely, we have

Theorem 1. For $p \in p_{n}$ and If $p(z)=a_{n} z^{n}+$ $\sum_{j=\mu}^{n} a_{n-j} z^{n-j}, 1 \leq \mu \leq n$ having no zeros in $|z|<k$, $k>0$, then for $0<r \leq R \leq k$, and $1 \leq s \leq n$

$$
\begin{aligned}
\max _{|z|=R}\left|p^{s}(z)\right| \leq & \frac{n(n-1) \cdots(n-s+1)}{R^{\mu}+k^{\mu}}\left(\frac{k^{\mu}+R^{\mu}}{k^{\mu}+r^{\mu}}\right)^{\frac{n}{\mu}} \\
& \left\{\max _{|z|=r}|p(z)|-\min _{|z|=k}|p(z)|\right\}
\end{aligned}
$$

The result is best possible for $s=1$ and equality holds for $p(z)=(z+k)^{n}$.

We now present some integral inequalities in the reverse direction for polynomials having a zero of order $s$ at the origin. More precisely, we prove

Theorem 2. Let $p \in p_{n}$ and $p(z)$ having all its zeros in $|z| \leq k, k \leq 1$ with a zero of order $s$ at $z=0$, then for $\beta$ with $|\beta|<k^{n-s}$ and $\gamma \geq 1$

$$
\left\|p^{\prime}-\frac{s m}{k^{n}} \bar{\beta} z^{s-1}\right\|_{\gamma} \geq\left\{n-(n-s) C_{\gamma}^{\mu}\left\|p+\frac{m}{k^{n}} \bar{\beta} z^{s}\right\|_{\gamma}\right\}
$$

Where $C_{\gamma}^{\mu}=\left\|\frac{k^{\mu}}{1+k^{\mu} z}\right\|_{\gamma}$.

By letting $\gamma \rightarrow \infty$ in place of Theorem 2, we obtain

Corollary 1. Let $p \in p_{n}$ and $p(z)$ having all its zeros in $|z| \leq k, k \leq 1$ with a zero of order $s$ at $z=0$, then for $\beta$ with $|\beta|<k^{n-s}$

$$
\left\|p^{\prime}(z)+\frac{s m}{k^{n}} \bar{\beta} z^{s-1}\right\|_{\infty} \geq\left(\frac{n+s k^{\mu}}{1+k^{\mu}}\right)\left\|p(z)+\frac{m}{k^{n}} \bar{\beta} z^{s}\right\|_{\infty} .
$$

For $\beta=0$ in inequality (2.3), we get

$$
\left\|p^{\prime}\right\|_{\infty} \geq\left(\frac{n+s k^{\mu}}{1+k^{\mu}}\right)\|p\|_{\infty}
$$

Next, we prove the following result which yields refinements of both the inequalities (1.8) and (1.9) as special cases.
Theorem 3. If $p(z)=a_{n} z^{n}+\sum_{j=\mu}^{n} a_{n-j} z^{n-j}, 1 \leq \mu \leq n$ having all its zeros in $|z| \leq k \leq 1$, with s-fold zeros at the origin, $0 \leq s \leq n$, then

$$
\begin{aligned}
\max _{|z|=1}\left|p^{\prime}(z)\right| \geq & \left(\frac{n+k^{\mu} s}{1+k^{\mu}}\right) \max _{|z|=1}|p(z)| \\
& +\frac{(n-s)}{k^{n-\mu}\left(1+k^{\mu}\right)} \min _{|z|=k}|p(z)| .
\end{aligned}
$$

The result is best possible and equality holds for $p(z)=$ $z^{s}(z+k)^{n-s}, 0 \leq s \leq n$.

\section{Lemmas}

For the proof of these theorems, we need the following lemmas.

Lemma 1. If $p(z)$ is a polynomial of degree $n$ having no zeros in $|z|<k, k \geq 1$, then

$$
\begin{aligned}
\max _{|z|=1}\left|p^{s}(z)\right| \leq & \frac{n(n-1) \cdots(n-s+1)}{1+k^{s}} \\
& \left\{\max _{|z|=1}|p(z)|-\min _{|z|=k}|p(z)|\right\} .
\end{aligned}
$$

Lemma 1 is due to Govil [7].

Lemma 2. If $p(z)=a_{0}+\sum_{j=\mu}^{n} a_{j} z^{j}, 1 \leq \mu \leq n$, is a polynomial of degree $n$ having no zeros in $|z|<k, k>0$, the for $0 \leq r \leq R \leq k$,

$$
\begin{aligned}
\max _{|z|=R}|p(z)| \leq & \left(\frac{k^{\mu}+R^{\mu}}{k^{\mu}+r^{\mu}}\right)^{\frac{n}{\mu}} \max _{|z|=1}|p(z)| \\
& +\left\{1-\left(\frac{k^{\mu}+R^{\mu}}{k^{\mu}+r^{\mu}}\right)^{\frac{n}{\mu}}\right\} \min _{|z|=k}|p(z)| .
\end{aligned}
$$

The above lemma is due to Dewan, Yadav and Pukhta $[5]$.

Lemma 3. If $p(z)=a_{0}+\sum_{j=\mu}^{n} a_{j} z^{j}, 1 \leq \mu \leq n$ is a polynomial of degree $n$ having all its zeros in $|z| \geq k \geq 1$ and $q(z)=z^{n} \overline{p\left(\frac{1}{\bar{z}}\right)}$, then for $|z|=1$,

$$
k^{\mu}\left|p^{\prime}(z)\right| \leq\left|q^{\prime}(z)\right|
$$

The above lemma is due to Chan and Malik [10]. By applying Lemma 3 to the polynomial one can deduce:

Lemma 4. If $p(z)=a_{n} z^{n}+\sum_{j=\mu}^{n} a_{n-j} z^{n-j}, 1 \leq \mu \leq n$, is a polynomial of degree $n$ having all its zeros in $|z| \leq$ $k \leq 1$ and $q(z)=z^{n} \overline{p\left(\frac{1}{\bar{z}}\right)}$, then for $|z|=1$,

$$
k^{\mu}\left|p^{\prime}(z)\right| \geq\left|q^{\prime}(z)\right| \text {. }
$$

Lemma 5. Let $p \in p_{n}$ and $p(z)=a_{0}+\sum_{j=\mu}^{n} a_{j} z^{j}$ having no zeros in $|z|<k, k \geq 1$, then for every complex number $\beta$ with $|\beta| \leq 1$ and for each $\gamma>0$,

$$
\left\|p^{\prime}(z)+\frac{m n}{1+k^{\mu}} \beta\right\|_{\gamma} \leq C_{\gamma}^{\mu}\|p\|_{\gamma} .
$$


Where $C_{\gamma}^{\mu}=\left\|\frac{k^{\mu}}{1+k^{\mu} z}\right\|_{\gamma}$.

The above lemma is due to Shah [11].

\section{Proof of Theorems}

Proof of Theorem 1. If $p(z)$ has no zeros in $|z|<k, k>$ 0 and if $0<r \leq R \leq k$, then $G(z)=P(R z)$ has no zeros in $|z|<\frac{k}{R}, \frac{k}{R}>1$.

Thus applying Lemma 1 to $G(z)$, we get

$$
\begin{aligned}
\max _{|z|=1}\left|G^{s}(z)\right| \leq & \frac{n(n-1) \cdots(n-s+1)}{1+\left(\frac{k}{R}\right)^{s}} \\
& \times\left\{\max _{|z|=1}|G(z)|-\min _{|z|=\frac{k}{R}}|G(z)|\right\}
\end{aligned}
$$

which implies

$$
\begin{aligned}
R^{s} \max _{|z|=1}\left|p^{s}(R z)\right| \leq & \frac{n(n-1) \cdots(n-s+1)}{1+\frac{k^{s}}{R^{s}}} \\
& \times\left\{\max _{|z|=1}|p(z)|-\min _{|z|=\frac{k}{R}}|p(R z)|\right\}
\end{aligned}
$$

which is equivalent to

$$
\begin{aligned}
\max _{|z|=R}\left|p^{\prime}(z)\right| \leq & \frac{n(n-1) \cdots(n-s+1)}{R^{s}+k^{s}} \\
& \times\left\{\max _{|z|=R}|p(z)|-\min _{|z|=k}|p(R z)|\right\}
\end{aligned}
$$

inequality (4.1) in conjunction with Lemma 2 yields,

$$
\begin{aligned}
\max _{|z|=R}\left|p^{s}(z)\right| \leq & \frac{n(n-1) \cdots(n-s+1)}{R^{s}+k^{s}}\left(\frac{k^{\mu}+R^{\mu}}{k^{\mu}+r^{\mu}}\right) \\
& \times\left\{\max _{|z|=r}|p(z)|-\min _{|z|=k}|p(z)|\right\} .
\end{aligned}
$$

This completes the proof of Theorem 1 .

Proof of Theorem 2. We have $p(z)=z^{s} \phi(z)$, where $\phi(z)$ is a polynomial of degree $n-s$, with the property that $\phi(0) \neq 0$, then

$$
q(z)=z^{n} \overline{p\left(\frac{1}{\bar{z}}\right)}=z^{n-s} \overline{\phi\left(\frac{1}{\bar{z}}\right)},
$$

is a polynomial of degree $n-s$ with no zeros in $|z|<\frac{1}{k}$. Now if $m_{0}=\min _{|z|=\frac{1}{k}}|q(z)|=\frac{m}{k^{n}}$.

By Rouche's theorem, the polynomial

$$
T(z)=q(z)+m_{0} \beta z^{n-s}, \quad|\beta|<k^{n-s}
$$

of degree $n-s$, will also have no zeros in $|z|<\frac{1}{k}, \frac{1}{k} \geq 1$. Hence by Lemma 5 , we have for $\gamma \geq 1$ and $|\beta|<k^{n-s}$

$$
\left\|T^{\prime}\right\|_{\gamma} \leq(n-s) C_{\gamma}^{\mu}\|T\|_{\gamma}
$$

or

$$
\begin{aligned}
& \left\|q^{\prime}(z)+\frac{(n-s) m}{k^{n}} \beta z^{(n-s-1)}\right\|_{\gamma} \\
& \quad \leq(n-s) C_{\gamma}^{\mu}\left\|q(z)+\frac{m}{k^{n}} \beta z^{n-s}\right\|_{\gamma}
\end{aligned}
$$

i.e.,

$$
\begin{gathered}
\left\|n p(z)-z p^{\prime}(z)+\frac{(n-s) m}{k^{n}} \bar{\beta} z^{s}\right\|_{\gamma} \\
\quad \leq(n-s) C_{\gamma}^{\mu}\left\|p(z)+\frac{m}{k^{n}} \bar{\beta} z^{s}\right\|_{\gamma} .
\end{gathered}
$$

Now by Minkowski inequality, we have for $\gamma \geq 1$ and $|\beta|<k^{n-s}$,

$$
\begin{aligned}
n\left\|p(z)+\frac{m}{k^{n}} \bar{\beta} z^{s}\right\|_{\gamma} \leq & \left\|n p(z)-z p^{\prime}(z)+\frac{(n-s) m}{k^{n}} \bar{\beta} z^{s}\right\|_{\gamma} \\
& +\left\|z p^{\prime}(z)+\frac{m S}{k^{n}} \bar{\beta} z^{s}\right\|_{\gamma} \\
\leq & (n-s) C_{\gamma}^{\mu}\left\|p(z)+\frac{m}{k^{n}} \bar{\beta} z^{s}\right\|_{\gamma} \\
& +\left\|z p^{\prime}(z)+\frac{m S}{k^{n}} \bar{\beta} z^{s}\right\|_{\gamma}
\end{aligned}
$$

which implies

$$
\begin{aligned}
& \left\|p^{\prime}(z)+\frac{s m}{k^{n}} \bar{\beta} z^{s-1}\right\| \\
& \quad \geq\left\{n-(n-s) C_{\nu}^{\mu}\left\|p+\frac{m}{k^{n}} \bar{\beta} z^{s}\right\|_{\gamma}\right\} .
\end{aligned}
$$

This completes the proof of Theorem 2 .

Proof of Theorem 3. Since all the zeros of $p(z)$ lie in $|z| \leq k \leq 1$, with $s$-fold zeros at the origin, therefore, for every complex number $\beta$ such that $|\beta|<1$, it follows by Rouche's theorem for $m>0$ that the polynomial $F(z)=p(z)-\frac{m}{k^{n}} \beta z^{n}$ has all its zero in $|z| \leq k, k \leq 1$. It can be easily verified that if $H(z)=z^{n} \overline{F\left(\frac{1}{\bar{z}}\right)}=z^{n-s} \overline{G\left(\frac{1}{\bar{z}}\right)}$, then

$$
\left|H^{\prime}(z)\right|=\left|n F(z)-z F^{\prime}(z)\right|, \quad \text { for }|z|=1 \text {. }
$$

Applying Lemma 4 to the polynomial $F(z)$, we get for $|z|=1$,

$$
k^{\mu}\left|F^{\prime}(z)\right| \geq\left|H^{\prime}(z)\right|=\left|n F(z)-z F^{\prime}(z)\right|
$$

Again applying inequality (2.4) to the polynomial $F(z)$, we get

$$
\left|F^{\prime}(z)\right| \geq \frac{n+s k^{\mu}}{1+k^{n}}|F(z)|, \quad \text { for }|z|=1 .
$$

Replacing $F(z)$ by $p(z)-\frac{\alpha m}{k^{n}} z^{n}$ in (4.2), we get

$$
\left|p^{\prime}(z)-\frac{\alpha n m}{k^{n}} z^{n-1}\right| \geq \frac{n+s k^{\mu}}{1+k^{\mu}}\left|p(z)-\frac{\alpha m}{k^{n}} z^{n}\right|,
$$

$$
\text { for }|z|=1
$$


for every $\alpha$ with $|\alpha|<1$. Choosing the argument of $\alpha$ such that

$$
\left|p(z)-\frac{\alpha m}{k^{n}} z^{n}\right|=|p(z)|-|\alpha| \frac{m}{k^{n}}, \quad \text { for }|z|=1 .
$$

It follows from (3.3) that

$$
\begin{array}{r}
\left|p^{\prime}(z)\right|-\frac{n|\alpha| m}{k^{n}} \geq \frac{n+s k^{\mu}}{1+k^{n}}\left\{|p(z)|-\frac{|\alpha| m}{k^{n}}\right\} \\
\text { for }|z|=1 .
\end{array}
$$

Letting $|\alpha| \rightarrow 1$, we obtain

$$
\left|p^{\prime}(z)\right| \geq \frac{n+s k^{\mu}}{1+k^{\mu}}|p(z)|+\left\{n-\frac{n+s k^{\mu}}{1+k^{\mu}}\right\} \frac{m}{k^{n}}
$$

this implies

$\max _{|z|=1}\left|p^{\prime}(z)\right| \geq \frac{n+s k^{\mu}}{1+k^{\mu}}|p(z)|+\frac{(n-s)}{k^{n-\mu}\left(1+k^{\mu}\right)} \min _{|z|=k}|p(z)|$.

This completes the proof of Theorem 3 .

\section{References}

[1] A. Aziz and Q.M. Dawood, Inequalities for a polynomial and its derivative, J. Approx. Theory, Vol. 54 (1988), 306-313.

[2] M. Bidkham and K.K. Dewan, Inequalities for a polynomial and its derivative, J. Math. Anal. Appl., Vol. 166 (1992), 319-324.

[3] N.K. Govil, On the derivative of a polynomial, Proc. Amer. Math. Soc., Vol. 41 (1973), 543-546.
[4] A. Aziz and W. M. Shah, Inequalities for a polynomial and its derivative, Math. Inequal. Appl., Vol. 7 (3) (2004), 379-391.

[5] K.K. Dewan, R.S. Yadav and M.S. Pukhta, Inequalities for a polynomial and its derivative, Math. Inequal. Appl., Vol. 2 (2) (1999), 203-205.

[6] N.K. Govil and G.N. McTume, Some generalization involving the polar derivatie of an inequality of Paul Turan, Acta Math Hungar, Vol. 104 (1-2) (2004), $115-126$.

[7] N.K. Govil, Some inequalities for derivatives of polynomials, J. Approx. Theory, Vol. 66 (1991), 2935 .

[8] M.A. Malik, On the derivative of a polynomial, J. London Math. Soc., Vol. (2) 1 (1969), 57-60.

[9] P. Turan, Uber die Ableitung von polynomena, Composito Math. Soc., Vol. 25 (1993), 49-54.

[10] T.N. Chan and M.A. Malik, On Erdös-Lax theorem, Proc. Indian Acad. Sci (Math. Sci), Vol. 92 (3) (1983), 191-193.

[11] W.M. Shah, Ph.D. Thesis submitted to University of Kashmir, India (1998).

[12] A. C. Schaeffer, Inequalities of A. Markoff and S. Bernstein for polynomials and related functions, Bull. Amer. Math. Soc., Vol. 47 (1941), 565-579.

[13] G.V. Milovanovico, D.S. Mitrinovic and Th. M. Rassias, Topics in Polynomial, Extremal properties, Inequalities and Zeros, World Scientific Publishing Company Co., Singapore, 1994. 